\title{
TITLE:
}

\section{On the symmetry of the linearized Boltzmann equation}

$\operatorname{AUTHOR}(\mathrm{S})$ :

Takata, Shigeru

CITATION:

Takata, Shigeru. On the symmetry of the linearized Boltzmann equation. AlP Conference Proceedings 2011, 1333: 49-56

\section{ISSUE DATE:}

2011

URL:

http://hdl.handle.net/2433/160665

\section{RIGHT:}

Copyright 2011 American Institute of Physics. This article may be downloaded for personal use only. Any other use requires prior permission of the author and the American Institute of Physics. The following article appeared in AIP Conference Proceedings 1333, 49 (2011) and may be found at http://link.aip.org/link/?apc/1333/49 


\title{
On the symmetry of the linearized Boltzmann equation
}

\author{
Shigeru TAKATA \\ Department of Mechanical Engineering and Science, also in Advanced Research Institute of Fluid Science and
Engineering, Graduate School of Engineering, Kyoto University, Kyoto 606-8501, Japan
}

\begin{abstract}
Relations between different problems described by the linearized Boltzmann equation (LBE) are discussed on the basis of the classical symmetry of LBE and kinetic boundary condition. The theory is developed for the system of arbitrary Knudsen number $(\mathrm{Kn})$ from the view point of the Green function. After showing the main idea of the approach to steady problems for bounded and unbounded domains, its extension to unsteady problems in a fixed bounded domain is given. In particular, in the latter, recovery of Kubo formula for the viscosity and thermal conductivity in the fluctuation-dissipation theorem and its extension to the systems of arbitrary Kn are shown. A comment on the extension to gas mixtures is also given.
\end{abstract}

Keywords: Boltzmann equation, kinetic theory, symmetry, Green reciprocity, Onsager-Casimir relation, fluctuation-dissipation theorem PACS: 51.10.+y, 47.45.-n, 47.61.Cb, 05.20.Dd, 05.70.Ln

\section{INTRODUCTION}

Relations between different problems described by the linearized Boltzmann equation have been discussed by many researchers because of the interest in Onsager-Casimir relation based on the entropy production argument (e.g., Refs. $[1,2,3,4,5,6,7,8,9,10])$. For example, Poiseuille flow vs. thermal transpiration and thermophoresis vs. thermal polarization are typical examples of such relations. In the present paper, we review the main results of our recent papers $[11,12,13]$ that are based on the idea of the Green function and give a direct interpretation of those relations without entropy production argument. The theory developed in these references makes use of the classical symmetry of the linearized collision operator and scattering kernel of the boundary condition [15, 5], complemented by the discussions on the far field behavior in the case of unbounded domain. The used symmetry is the standard property for the kinetic theory description to be consistent with the second law of thermodynamics [5]. The reciprocal relations between different problems can be understood by the presented approach as a consequence of the Green reciprocity. Different from the entropy production argument, the thin layer that contains the surface of (or the interface with) the body inside is not included in the considered domain in our theory [11,12]. This feature enables us to develop the theory for time-dependent problems [12] as a natural extension from the steady case [11]. As a consequence, a reinterpretation of the Kubo formulas (the fluctuation-dissipation theorem) for the viscosity and thermal conductivity is obtained. Further, mass and heat flows through a channel can be understood as a generalization of those formulas.

We shall show below the above mentioned results, following Refs. [11, 12]. However, we would like the reader to remember that some physical settings and notations here may be changed from the originals $[11,12]$ for conciseness. Poiseuille flow and thermal transpiration will be repeated in the sequel as an illustrative example.

\section{SYMMETRIC RELATION FOR STEADY BOUNDARY-VALUE PROBLEMS}

Consider the steady behavior of a single-component monatomic rarefied gas in a domain $\mathscr{D}$, where $\mathscr{D}$ may be bounded or unbounded. The state of the gas is so close to the reference equilibrium state at rest with uniform density $\rho_{0}$ and temperature $T_{0}$ that the second (or higher) order effect of the deviation from this state can be neglected (linearization).

With a proper choice of reference length $L$, we denote the spatial coordinates, molecular velocity, and velocity distribution function of molecules by $L \boldsymbol{x},\left(2 k T_{0} / m\right)^{1 / 2} \boldsymbol{\zeta}$, and $\rho_{0}\left(2 k T_{0} / m\right)^{-3 / 2}[1+\boldsymbol{\phi}(\boldsymbol{x}, \boldsymbol{\zeta})] E(\boldsymbol{\zeta})$. Here $k$ is the Boltzmann constant, $m$ is the mass of a molecules, and $E(\boldsymbol{\zeta})=\pi^{-3 / 2} \exp \left(-|\zeta|^{2}\right)$. Denoting by $D$ the region of $\boldsymbol{x}$ corresponding to the dimensional region $\mathscr{D}$, the gas behavior is described by the following steady linearized Boltzmann equation:

$$
\zeta_{i} \frac{\partial \phi}{\partial x_{i}}=\frac{2}{\sqrt{\pi} \mathrm{Kn}} \mathscr{L}(\phi)+I(\boldsymbol{x}, \boldsymbol{\zeta}), \quad\left(\boldsymbol{x} \in D, \zeta \in \mathbb{R}^{3}\right) .
$$

27th International Symposium on Rarefied Gas Dynamics, 2010

AIP Conf. Proc. 1333, 49-56 (2011); doi: 10.1063/1.3562624

(C) 2011 American Institute of Physics 978-0-7354-0888-0/\$30.00 
Here $\mathrm{Kn}$ is the Knudsen number $\left(0<\mathrm{Kn}<\infty ; \mathrm{Kn}=\ell_{0} / L\right.$ with $\ell_{0}$ being the mean free path at the reference state) and $I$ is a given function. Most typically, $I$ represents the effect of a weak external force, though it is not limited to some real force (thus $I$ will be referred to "external force" in the sequel). $\mathscr{L}$ has the following well-known properties [15, 16]:

(i) $\mathscr{L}(\Phi)^{-}=\mathscr{L}\left(\Phi^{-}\right)$, where $\Phi^{-}(\boldsymbol{x}, \boldsymbol{\zeta}) \equiv \Phi(\boldsymbol{x},-\boldsymbol{\zeta})(\mathscr{L}$ commutes with symmetries in $\boldsymbol{\zeta}$-space),

(ii) $\langle\Phi \mathscr{L}(\Psi)\rangle=\langle\Psi \mathscr{L}(\Phi)\rangle$, where $\langle\ldots\rangle=\int \ldots(\boldsymbol{\zeta}) E(\boldsymbol{\zeta}) \mathrm{d} \zeta$ (self-adjointness),

(iii) $\mathscr{L}(\Phi)=0$ holds if and only if $\Phi$ is a linear combination of $1, \zeta$, and $|\zeta|^{2}$,

(iv) $\langle\Phi \mathscr{L}(\Phi)\rangle \leq 0$ and the equality holds if and only if $\Phi$ is a linear combination of $1, \zeta$, and $|\zeta|^{2}$ ( $\mathscr{L}$ is non-negative).

The boundary $\partial D$ of the domain $D$ is classified into two parts: the interface with or surface of non-gas material ("body"), say real boundary $\partial D_{\mathrm{w}}$, and artificial boundary $\partial D_{\mathrm{g}}$, i.e., $\partial D=\partial D_{\mathrm{w}} \cup \partial D_{\mathrm{g}} .{ }^{1} \phi$ obeys the following condition on $\partial D_{\mathrm{w}}$ :

$$
\begin{gathered}
\phi=g_{\mathrm{w}}+\int_{\zeta_{i}^{*} n_{i}<0} \frac{\left|\zeta_{i}^{*} n_{i}\right| E\left(\boldsymbol{\zeta}^{*}\right)}{\left|\zeta_{i} n_{i}\right| E(\boldsymbol{\zeta})} R\left(\boldsymbol{\zeta}^{*}, \zeta ; \boldsymbol{x}\right)\left(\boldsymbol{\phi}^{*}-g_{\mathrm{w}}^{*}\right) \mathrm{d} \boldsymbol{\zeta}^{*}, \quad \zeta_{i} n_{i}>0 \\
g_{\mathrm{w}}(\boldsymbol{x}, \boldsymbol{\zeta})=P_{\mathrm{w}}(\boldsymbol{x})+2 \zeta_{i} u_{\mathrm{w} i}(\boldsymbol{x})+\left(|\boldsymbol{\zeta}|^{2}-\frac{5}{2}\right) \tau_{\mathrm{w}}(\boldsymbol{x})
\end{gathered}
$$

where $\phi$ and $g_{\mathrm{w}}$ with $*$ indicate that their argument $\boldsymbol{\zeta}$ is replaced by $\boldsymbol{\zeta}^{*} ; \boldsymbol{n}$ (or $n_{i}$ ) is the unit inward normal to the boundary; and $\left(2 R T_{0}\right)^{1 / 2} \boldsymbol{u}_{\mathrm{w}}, T_{0}\left(1+\tau_{\mathrm{w}}\right)$, and $p_{0}\left(1+P_{\mathrm{w}}\right)$ denote the velocity of the body surface, its temperature, and corresponding saturation pressure of the gas $\left(p_{0}=\rho_{0} k T_{0} / m\right)$. Because of the steady problem, $D$ does not deform, so that $u_{\mathrm{w} i} n_{i}=0 . R$ represents the scattering law for gas molecules on the boundary at the reference state. $R$ should satisfy standard properties in kinetic theory (such as the uniqueness condition [16], detailed balance [15, 5]), though there are some differences depending on whether $\partial D_{\mathrm{w}}$ is a simple boundary or condensed phase. The reader is referred to Appendix of Ref. [12] for the details. Here, we just remark that, when $\partial D_{\mathrm{w}}$ is a simple boundary, $\left\langle\zeta_{i} n_{i} \phi\right\rangle=0$ and consequently $P_{\mathrm{w}}$ may be replaced by an arbitrary constant (for example, one may put $P_{\mathrm{w}}=0$ ).

Next consider the imaginary boundary $\partial D_{\mathrm{g}}$. We consider the imaginary boundary composed, in the most general case, of the following three parts $\left(\partial D_{\mathrm{g}}=\partial D_{\mathrm{g}}^{(1)} \cup \partial D_{\mathrm{g}}^{(2)} \cup \partial D_{\mathrm{g}}^{(3)}\right)$, where $\partial D_{\mathrm{g}}^{(3)}$ represents the boundary at infinity when $D$ is unbounded:

(i) On $\partial D_{\mathrm{g}}^{(1)}, \phi$ obeys

$$
\phi(\boldsymbol{x}, \boldsymbol{\zeta})=h_{\mathrm{in}}(\boldsymbol{x}, \boldsymbol{\zeta}) \text { for } \zeta_{i} n_{i}>0, \boldsymbol{x} \in \partial D_{\mathrm{g}}^{(1)},
$$

where $h_{\text {in }}$ is a given function for $\zeta_{i} n_{i}>0$. For later discussions, we denote this function extended to the whole range of $\zeta$ by $h(\boldsymbol{x}, \boldsymbol{\zeta})$. The way of extension is arbitrary and does not influence the results that follow.

(ii) On $\partial D_{\mathrm{g}}^{(2)}, \phi$ obeys

$$
\phi=h(\boldsymbol{x}, \boldsymbol{\zeta})+\int_{\partial D_{\mathrm{g}}^{(2)}} \int_{\zeta_{i}^{\prime} n_{i}^{\prime}<0} P\left(\boldsymbol{x}^{\prime}, \boldsymbol{\zeta}^{\prime}, \boldsymbol{x}, \boldsymbol{\zeta}\right)\left(\phi^{\prime}-h^{\prime}\right) \mathrm{d} \boldsymbol{\zeta}^{\prime} \mathrm{d} S^{\prime} \quad \text { for } \zeta_{i} n_{i}>0, \boldsymbol{x} \in \partial D_{\mathrm{g}}^{(2)},
$$

where $h(\boldsymbol{x}, \boldsymbol{\zeta})$ is a given function for $\boldsymbol{\zeta} \in \mathbb{R}^{3}, \boldsymbol{x} \in \partial D_{\mathrm{g}}^{(2)} ; \boldsymbol{n}^{\prime}$ is the inward unit vector normal to $\partial D_{\mathrm{g}}^{(2)}$ at position $\boldsymbol{x}^{\prime} ; \mathrm{dS} \mathrm{S}^{\prime}$ is the surface element of $\partial D_{\mathrm{g}}^{(2)}$ at position $\boldsymbol{x}^{\prime} ;$ and $\phi^{\prime}=\phi\left(\boldsymbol{x}^{\prime}, \boldsymbol{\zeta}^{\prime}\right)$ and $h^{\prime}=h\left(\boldsymbol{x}^{\prime}, \boldsymbol{\zeta}^{\prime}\right)$.

(iii) When $D$ is unbounded, $\phi$ obeys the following condition at infinity (i.e., $\partial D_{\mathrm{g}}^{(3)}$ ):

$$
\phi \rightarrow h(\boldsymbol{x}, \boldsymbol{\zeta}) \quad \text { as }|\boldsymbol{x}| \rightarrow \infty,
$$

where $h(\boldsymbol{x}, \boldsymbol{\zeta})$ is a solution of the Boltzmann equation (1).

The reader is referred to Appendix of Ref. [12] for the properties of $P$ on $\partial D_{\mathrm{g}}^{(2)}$. Here we just remark that the typical example of $P$ on $\partial D_{\mathrm{g}}^{(2)}$ is that $\phi-h$ obeys the specular reflection or periodic condition. The most important is that the following relations hold on the boundary:

$$
\left\langle\zeta_{i} n_{i}\left(\phi-g_{\mathrm{w}}\right)\left(\phi^{-}-g_{\mathrm{w}}^{-}\right)\right\rangle=0 \quad \text { on } \partial D_{\mathrm{w}}, \quad \int_{\partial D_{\mathrm{g}}^{(1)} \cup \partial D_{\mathrm{g}}^{(2)}}\left\langle\zeta_{i} n_{i}(\phi-h)\left(\phi^{-}-h^{-}\right)\right\rangle \mathrm{dS}=0
$$

\footnotetext{
${ }^{1}$ For example, $\partial D_{\mathrm{g}}$ is some control surface taken in a gas. Specular reflection and periodic boundary will also be classified into $\partial D_{\mathrm{g}}$.
} 
Now we are ready to state the symmetric relation for steady problems:

Proposition 1 Consider the solutions $\phi^{A}$ and $\phi^{B}$ of the boundary-value problem (1)-(3) such that $\phi^{A}$ (or $\left.\phi^{B}\right)$ is a solution in the case of $I=I^{A}, g_{\mathrm{w}}=g_{\mathrm{w}}^{A}$, and $h=h^{A}\left(\right.$ or $I=I^{B}, g_{\mathrm{w}}=g_{\mathrm{w}}^{B}$, and $\left.h=h^{B}\right)$, where $\mathrm{Kn}(0<\mathrm{Kn}<\infty), R$ in (2a), and $P$ in (3b) are common to the problems. Then, if $\partial D_{\mathrm{g}}^{(3)}$ is absent or $\phi^{A}$ and $\phi^{B}$ approach $h^{A}$ and $h^{B}$ in such a way that

$$
\int_{\partial D_{\mathrm{g}}^{(3)}}\left\langle\zeta_{i} n_{i}\left(\phi^{A-}-h^{A-}\right)\left(\phi^{B}-h^{B}\right)\right\rangle \mathrm{dS}=0
$$

the following equality holds:

$$
\begin{aligned}
& \int_{\partial D_{\mathrm{w}}}\left\langle\zeta_{i} n_{i} g_{\mathrm{w}}^{B-} \phi^{A}\right\rangle \mathrm{d} \mathrm{S}+\int_{\partial D_{\mathrm{g}}}\left\langle\zeta_{i} n_{i} h^{B-} \phi^{A}\right\rangle \mathrm{d} \mathrm{S}-\frac{1}{2} \int_{\partial D_{\mathrm{g}}}\left\langle\zeta_{i} n_{i} h^{B-} h^{A}\right\rangle \mathrm{d} \mathrm{S}-\int_{D}\left\langle I^{B-} \phi^{A}\right\rangle \mathrm{d} \boldsymbol{x} \\
= & \int_{\partial D_{\mathrm{w}}}\left\langle\zeta_{i} n_{i} g_{\mathrm{w}}^{A-} \phi^{B}\right\rangle \mathrm{d} \mathrm{S}+\int_{\partial D_{\mathrm{g}}}\left\langle\zeta_{i} n_{i} h^{A-} \phi^{B}\right\rangle \mathrm{d} \mathrm{S}-\frac{1}{2} \int_{\partial D_{\mathrm{g}}}\left\langle\zeta_{i} n_{i} h^{A-} h^{B}\right\rangle \mathrm{d} \mathrm{S}-\int_{D}\left\langle I^{A-} \phi^{B}\right\rangle \mathrm{d} \boldsymbol{x} .
\end{aligned}
$$

It should be noted that the relation (6) holds unconditionally for bounded domain, while it is not clear whether or not condition (5) for (6) to hold is fulfilled in general for unbounded domain. This is due to the slow decay of $\phi-h$ at a far distance. ${ }^{2}$ Nevertheless,

1. for spatially one dimensional half-space problems,

2. for the case where $\partial D_{\mathrm{w}}$ is confined in a finite ball,

we can prove (5) and thus the relation (6) (see lemma 2 of Ref. [11]). For the other cases, one is required to check the condition (5) in each application of (6). Clarifying a general situation for (5) to hold would be desired.

In the relation (6), the role of problems A and B is symmetric. One of the important features of (6) is that each term is a definite flux of $\phi^{A}$ or $\phi^{B}$. It is due to (4) and (5) and implies that a proper flux through the boundary of concerned problem may be obtained from a flux of another problem. This observation eventually leads us to a general representation of flux through the boundary in terms of a properly defined Green function, which we shall explain in the next section. The relations between independent problems mentioned in the introduction are to be obtained as a consequence of that representation $[11,13]$. Another feature of $(6)$ is that $I$ may be arbitrary and is not limited to the one arising from the linearization around local Maxwellian such as $\zeta_{i}$ or $\zeta_{i}\left(|\zeta|^{2}-\frac{5}{2}\right)$. Actually, some unsteady problems and steady half-space problems can be formulated as steady problems with other forms of $I$. Thus, for instance, relations between second-order slip coefficients can be discussed by the relation (6) (see Sec. 3 of Ref. [11]). ${ }^{3}$

\section{GREEN FUNCTION AND GENERAL REPRESENTATION FOR FLUXES}

In this section, we introduce the Green function and discuss the general representation for the flux. Here we restrict ourselves to the simplest case where $D$ is bounded, $\partial D=\partial D_{\mathrm{w}}$, and $I=0$, for the sake of clarity. ${ }^{4}$

Taking into account the specific form of $g_{\mathrm{w}}$, let us consider the response of the system to the point source $\alpha$ placed at $\boldsymbol{x}_{0}$ on $\partial D_{\mathrm{w}}$ and denote it by $G^{\left(\alpha ; \boldsymbol{x}_{0}\right)}(\boldsymbol{x}, \boldsymbol{\zeta})$, where $\alpha=1,2 \zeta_{i} t_{i}\left(\boldsymbol{x}_{0}\right),|\boldsymbol{\zeta}|^{2}-\frac{5}{2}$ and $t_{i}\left(\boldsymbol{x}_{0}\right)$ is a unit tangential vector to $\partial D_{\mathrm{w}}$ at $\boldsymbol{x}_{0}$. Thus, $G^{\left(\alpha ; x_{0}\right)}(\boldsymbol{x}, \boldsymbol{\zeta})$ is a solution of the boundary-value problem (1) and (2) with $I=0$ and $g_{\mathrm{w}}=\alpha \delta\left(\boldsymbol{x}-\boldsymbol{x}_{0}\right)$. Applying the symmetric relation (6) to the pair of $G^{\left(\alpha ; x_{0}\right)}(\boldsymbol{x}, \boldsymbol{\zeta})$ and $G^{\left(\beta ; x_{1}\right)}(\boldsymbol{x}, \boldsymbol{\zeta})\left(\beta=1,2 \zeta_{i} t_{i}\left(\boldsymbol{x}_{1}\right),|\boldsymbol{\zeta}|^{2}-\frac{5}{2}\right)$ leads to

$$
\left\langle\zeta_{i} n_{i} \beta^{-} G^{\left(\alpha ; x_{0}\right)}\right\rangle\left(x_{1}\right)=\left\langle\zeta_{i} n_{i} \alpha^{-} G^{\left(\beta ; x_{1}\right)}\right\rangle\left(x_{0}\right) .
$$

\footnotetext{
${ }^{2}$ Note that, in three dimensional problem, according to Fourier's law of heat conduction and Stokes equation for incompressible fluid, temperature and flow velocity approach their far field with the rate of $1 /|\boldsymbol{x}|$ if $\partial D_{\mathrm{w}}$ is confined in a finite ball. Such behavior is properly discussed in Ref. [6], while much faster approach is claimed in Ref. [7] by assuming the local Maxwellian at a far distance. As pointed out in Refs. [11, 14], the fast approach estimate in Ref. [7] is not correct. Incidentally, there is no estimate on the far field behavior in Ref. [10].

3 In some problems, both side of (6) diverge due to the form of $I$. In many cases, however, such divergence can be avoided by a proper transformation. In the reformulated problem, $g_{\mathrm{w}}$ may be changed to a more general form. In the case, in applying Proposition $\left.1,-\frac{1}{2} \int_{\partial D_{\mathrm{w}}}\left\langle\zeta_{i} n_{i} g_{\mathrm{w}}^{B-} g_{\mathrm{w}}^{A}\right\rangle\right\rangle \mathrm{dS}$ should be added to the left-hand side and $\left.-\frac{1}{2} \int_{\partial D_{\mathrm{w}}}\left\langle\zeta_{i} n_{i} g_{\mathrm{w}}^{A-} g_{\mathrm{w}}^{B}\right\rangle\right\rangle \mathrm{dS}$ to the right-hand side of (6).

${ }^{4}$ Ref. [11] gives a unified argument for two cases under $I=0$ : (i) $\partial D_{\mathrm{g}}=\partial D_{\mathrm{g}}^{(1)} \cup \partial D_{\mathrm{g}}^{(2)}$ (thus $D$ is bounded) and (ii) $\partial D_{\mathrm{g}}=\partial D_{\mathrm{g}}^{(3)}$ (thus $D$ is unbounded).
} 


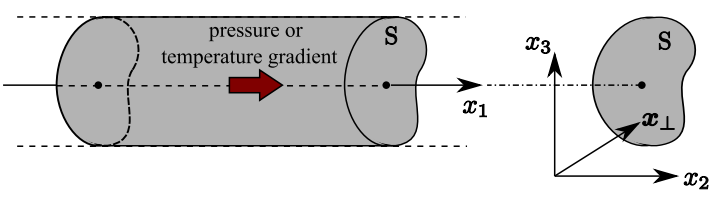

(a)

(b)

FIGURE 1. Poiseuille and thermal transpiration flows. (a) Sketch of the problems. (b) Geometry of the pipe cross-section.

This means that the cause and result (induced flow or flux) are reciprocal between two different points on the boundary (Green reciprocity). Note that the solution of the same problem with arbitrary $g_{\mathrm{w}}($ and $I=0)$ can be expressed by

$$
\phi(\boldsymbol{x}, \boldsymbol{\zeta})=\int_{\partial D_{\mathrm{w}}}\left(P_{\mathrm{w}}\left(\boldsymbol{x}_{0}\right) G^{\left(1 ; \boldsymbol{x}_{0}\right)}(\boldsymbol{x}, \boldsymbol{\zeta})+\left|\boldsymbol{u}_{\mathrm{w}}\left(\boldsymbol{x}_{0}\right)\right| G^{\left(2 \zeta_{i} \hat{u}_{\mathrm{w} i} ; \boldsymbol{x}_{0}\right)}(\boldsymbol{x}, \boldsymbol{\zeta})+\tau_{\mathrm{w}}\left(\boldsymbol{x}_{0}\right) G^{\left(|\boldsymbol{\zeta}|^{2}-\frac{5}{2} ; \boldsymbol{x}_{0}\right)}\right) \mathrm{d} \mathrm{S}_{0},
$$

where $\mathrm{dS}_{0}$ denotes the element surface at position $\boldsymbol{x}_{0}$ and $\hat{\boldsymbol{u}}_{\mathrm{W}}$ is the unit vector in the direction of $\boldsymbol{u}_{\mathrm{w}}$. Thus, by taking into account (7), the mass, momentum, and heat flows across the boundary $\partial D_{\mathrm{w}}$ at $\boldsymbol{x}_{1}$ is expressed as

$$
\left\langle\zeta_{i} n_{i} \beta^{-} \phi\right\rangle\left(\boldsymbol{x}_{1}\right)=\int_{\partial D_{\mathrm{w}}}\left(P_{\mathrm{w}}\left\langle\zeta_{i} n_{i} G^{\left(\beta ; \boldsymbol{x}_{1}\right)}\right\rangle-\boldsymbol{u}_{\mathrm{w} j}\left\langle 2 \zeta_{i} \zeta_{j} n_{i} G^{\left(\beta ; \boldsymbol{x}_{1}\right)}\right\rangle+\tau_{\mathrm{w}}\left\langle\zeta_{i} n_{i}\left(|\boldsymbol{\zeta}|^{2}-\frac{5}{2}\right) G^{\left(\beta ; \boldsymbol{x}_{1}\right)}\right\rangle\right) \mathrm{d} \mathrm{S}
$$

where $\beta=1,2 \zeta_{i} t_{i}\left(\boldsymbol{x}_{1}\right)$, and $|\boldsymbol{\zeta}|^{2}-\frac{5}{2}$ gives mass-, momentum-, and heat-flow expression. This is the general representation of the fluxes through the boundary in the simplest case. Equation (8) shows that the flow (or flux) of $\beta$-moment at $\boldsymbol{x}_{1}$ on $\partial D_{\mathrm{w}}$ is immediately obtained if one knows the system response to the point source of $\beta$ at $\boldsymbol{x}_{1}$. If (8) is integrated with respect to $x_{1}$ over a part of boundary $S\left(\subset \partial D_{\mathrm{w}}\right)$, the mass and heat fluxes through $S$ is obtained as

$$
\int_{S}\left\langle\zeta_{i} n_{i} \beta^{-} \phi\right\rangle \mathrm{d} \mathrm{S}=\int_{\partial D_{\mathrm{w}}}\left(P_{\mathrm{w}}\left\langle\zeta_{i} n_{i} G^{(\beta ; S)}\right\rangle-\boldsymbol{u}_{\mathrm{w} j}\left\langle 2 \zeta_{i} \zeta_{j} n_{i} G^{(\beta ; S)}\right\rangle+\tau_{\mathrm{w}}\left\langle\zeta_{i} n_{i}\left(|\boldsymbol{\zeta}|^{2}-\frac{5}{2}\right) G^{(\beta ; S)}\right\rangle\right) \mathrm{d} \mathrm{S},
$$

where $\beta=1,|\boldsymbol{\zeta}|^{2}-\frac{5}{2}$ and $G^{(\alpha ; S)}(\boldsymbol{x}, \boldsymbol{\zeta})$ is a solution of the boundary-value problem (1) and (2) with $I=0$ and $g_{\mathrm{w}}=\alpha \chi_{S}(\boldsymbol{x})$, where $\chi_{S}(\boldsymbol{x})=1$ if $\boldsymbol{x} \in S$ and $\chi_{S}(\boldsymbol{x})=0$ otherwise.

Discussions of the above idea for a wider class of situations can be found in Ref. [11] (see footnote 4). For example, if $D$ is bounded and a part of its boundary is artificial, the fluxes through some part $S \subset \partial D_{\mathrm{g}}$ are expressed as

$$
\int_{S}\left\langle\zeta_{i} n_{i} \alpha^{-} \phi\right\rangle \mathrm{d} \mathrm{S}=\int_{\partial D_{\mathrm{w}}}\left\langle\zeta_{i} n_{i} g_{\mathrm{w}}^{-} G^{(\alpha, S)}\right\rangle \mathrm{d} \mathrm{S}+\int_{\partial D_{\mathrm{g}}}\left\langle\zeta_{i} n_{i} h^{-} G^{(\alpha, S)}\right\rangle \mathrm{d} \mathrm{S}+\int_{S}\left\langle\zeta_{i} n_{i} \alpha^{-} h\right\rangle \mathrm{dS},
$$

where $\alpha=1,2 \zeta_{j},|\zeta|^{2}-\frac{5}{2}$ and $G^{(\alpha ; S)}(\boldsymbol{x}, \boldsymbol{\zeta})$ is a solution of the boundary-value problem (1)-(3b) with $I=g_{\mathrm{w}}=0$ and $h=\alpha \chi_{S}(\boldsymbol{x})$. The reader is referred to Secs. 4 and 5 of Ref. [11] for more general representations and examples.

Poiseuille flow and thermal transpiration. Consider the steady Poiseuille flow and thermal transpiration in a straight pipe (Fig. 1). The geometry of the pipe cross-section may be arbitrary. Let us denote the perturbed velocity distribution function of the former by $\phi^{\mathrm{P}}(\boldsymbol{x}, \boldsymbol{\zeta})$ and the latter by $\phi^{\mathrm{T}}(\boldsymbol{x}, \boldsymbol{\zeta})$. Then $\phi^{\mathrm{P}}$ is a solution of (1), (2), and (3b) in the domain grayed in Fig. $1\left[0 \leq x_{1} \leq 1\right.$ and $\boldsymbol{x}_{\perp} \in \mathrm{S}$, where $\left.\boldsymbol{x}_{\perp}=\left(x_{2}, x_{3}\right)\right]$ with $g_{\mathrm{w}}=I=0$ and $h=x_{1}$, while $\phi^{\mathrm{T}}$ is a solution of (1), (2), and (3b) in the same domain with $g_{\mathrm{w}}=h=x_{1}\left(|\zeta|^{2}-\frac{5}{2}\right)$ and $I=0$ (magnitude of imposed gradient of pressure and temperature are rescaled as unity because of the linear problem; $P$ is the kernel of periodic condition). Note that $\phi^{\mathrm{P}}$ is the Green function $G^{(1 ; \mathrm{S})}$ of the considered domain. Thus, from (10) with $\alpha=1$, the following expression is eventually obtained (by taking account of obvious properties of $\phi^{\mathrm{P}}$ and $\phi^{\mathrm{T}}$ ):

$$
\int_{\mathrm{S}}\left\langle\zeta_{1} \phi^{\mathrm{T}}\right\rangle \mathrm{dS}=\int_{\mathrm{S}}\left\langle\zeta_{1}\left(|\zeta|^{2}-\frac{5}{2}\right) \phi^{\mathrm{P}}\right\rangle \mathrm{dS}
$$

This is the known reciprocity relation [3] as the Onsager-Casimir relation (see also Example 5 of Ref. [11]).

We finally mention that the relation of the above theory to entropy production argument is discussed in Ref. [14]. In Ref. [14], it is shown that the conventional Onsager-Casimir relation can be established in a pointwise way, thanks to the Green reciprocity. Further, the situation where the conventional Onsager-Casimir relation holds is clarified. ${ }^{5}$

\footnotetext{
${ }^{5}$ It is pointed out from early days that the conventional Onsager-Casimir relation does not hold in general, even for gross quantities like the fluxes discussed above. See, e.g., Sec. 15.10 of Ref. [5] and references therein. Thermophoresis is an example [6, 13, 14].
} 


\section{SYMMETRIC RELATION AND GREEN FUNCTION FOR UNSTEADY PROBLEMS}

In discussing the reciprocity based on entropy production, it is necessary to consider the production in a thin layer that contains the interface between the gas and "body." The production in the layer is estimated indirectly from the entropy balance at steady state by assuming the local equilibrium inside the body, which has naturally limited the argument to steady problems. On the other hand, the symmetric relation and Green function approach in previous sections do not contain the thin layer in the considered domain. In other words, the argument of the thin layer is not necessary to discuss the relations between two independent problems, once one accepts boundary conditions that have the standard properties in kinetic theory. ${ }^{6}$ Thus, the approach in Ref. [11] can be extended to unsteady problems. In this section, we shall present some consequences obtained from this extension on the basis of Ref. [12].

With a proper reference scale $t_{0}$, we denote the time by $t_{0} t$. Below, $\phi, I, g_{\mathrm{w}}$ (or $P_{\mathrm{w}}, \boldsymbol{u}_{\mathrm{w}}$, and $\tau_{\mathrm{w}}$ ), $h_{\mathrm{in}}$, and $h$ may depend on time: $\phi(t, \boldsymbol{x}, \boldsymbol{\zeta}), I(t, \boldsymbol{x}, \boldsymbol{\zeta}), g_{\mathrm{w}}(t, \boldsymbol{x}, \boldsymbol{\zeta})\left[P_{\mathrm{w}}(t, \boldsymbol{x}), \boldsymbol{u}_{\mathrm{w}}(t, \boldsymbol{x})\right.$, and $\left.\tau_{\mathrm{w}}(t, \boldsymbol{x})\right], h_{\mathrm{in}}(t, \boldsymbol{x}, \boldsymbol{\zeta})$, and $h(t, \boldsymbol{x}, \boldsymbol{\zeta})$. However, the domain $D$ is bounded and does not change in time, so that $u_{\mathrm{w} i} n_{i}=0$. Then, the behavior of the gas is described by the following linearized Boltzmann equation in place of (1):

$$
\operatorname{Sh} \frac{\partial \phi}{\partial t}+\zeta_{i} \frac{\partial \phi}{\partial x_{i}}=\frac{2}{\sqrt{\pi}} \frac{1}{\mathrm{Kn}} \mathscr{L}(\phi)+I \quad\left(\zeta \in \mathbb{R}^{3}, \boldsymbol{x} \in D, t>0\right) .
$$

Here $\operatorname{Sh}\left[=L / t_{0}\left(2 k T_{0} / m\right)^{1 / 2}\right]$ is the Strouhal number. The initial data is denoted by putting the subscripted "initial":

$$
\phi(0, \boldsymbol{x}, \boldsymbol{\zeta})=\phi_{\text {initial }}(\boldsymbol{x}, \boldsymbol{\zeta}) \quad\left(\boldsymbol{\zeta} \in \mathbb{R}^{3}, \boldsymbol{x} \in D\right) .
$$

Since $D$ is bounded, $\partial D_{\mathrm{g}}^{(3)}$ does not exist. The kernels $R$ on $\partial D_{\mathrm{w}}$ and $P$ on $\partial D_{\mathrm{g}}^{(2)}$ do not depend on $t$. Then, for solutions of initial- and boundary-value problems (12), (13), (2)-(3b), we obtain the following:

Proposition 2 Let $\phi^{A}$ be a solution of the initial-and boundary-value problem (12), (13), (2)-(3b) with $I=I^{A}$, $g_{\mathrm{w}}=g_{\mathrm{w}}^{A}, h=h^{A}$, and $\phi_{\text {initial }}=\phi_{\text {initial }}^{A}$. Let $\phi^{B}$ be a solution of the initial-and boundary-value problem (12), (13), (2)-(3b) with $I=I^{B}, g_{\mathrm{w}}=g_{\mathrm{w}}^{B}, h=h^{B}$, and $\phi_{\text {initial }}=\phi_{\text {initial. }}^{B}$. Here the bounded domain D, the Strouhal and Knudsen numbers $\mathrm{Sh}$ and $\mathrm{Kn}$, the collision operator $\mathscr{L}$, and the kernels $R$ and $P$ are common to both the problems with solutions $\phi^{A}$ and $\phi^{B}$. Then, the following relation holds:

$$
\begin{aligned}
\mathrm{Sh} \int_{D}\left\langle\phi_{\text {initial }}^{B-} \phi^{A}\right\rangle(t, \boldsymbol{x}) \mathrm{d} \boldsymbol{x} & +\int_{D}\left\langle I^{B-} * \phi^{A}\right\rangle(t, \boldsymbol{x}) \mathrm{d} \boldsymbol{x}-\int_{\partial D_{\mathrm{w}}}\left\langle\zeta_{i} n_{i}\left(g_{\mathrm{w}}^{B-} * \phi^{A}\right)\right\rangle(t, \boldsymbol{x}) \mathrm{dS} \\
& -\int_{\partial D_{\mathrm{g}}}\left\langle\zeta_{i} n_{i}\left(h^{B-} * \phi^{A}\right)\right\rangle(t, \boldsymbol{x}) \mathrm{dS}+\frac{1}{2} \int_{\partial D_{\mathrm{g}}}\left\langle\zeta_{i} n_{i}\left(h^{B-} * h^{A}\right)\right\rangle(t, \boldsymbol{x}) \mathrm{d} \mathrm{S} \\
=\mathrm{Sh} \int_{D}\left\langle\phi_{\text {initial }}^{A-} \phi^{B}\right\rangle(t, \boldsymbol{x}) \mathrm{d} \boldsymbol{x} & +\int_{D}\left\langle I^{A-} * \phi^{B}\right\rangle(t, \boldsymbol{x}) \mathrm{d} \boldsymbol{x}-\int_{\partial D_{\mathrm{w}}}\left\langle\zeta_{i} n_{i}\left(g_{\mathrm{w}}^{A-} * \phi^{B}\right)\right\rangle(t, \boldsymbol{x}) \mathrm{dS} \\
& -\int_{\partial D_{\mathrm{g}}}\left\langle\zeta_{i} n_{i}\left(h^{A-} * \boldsymbol{\phi}^{B}\right)\right\rangle(t, \boldsymbol{x}) \mathrm{dS}+\frac{1}{2} \int_{\partial D_{\mathrm{g}}}\left\langle\zeta_{i} n_{i}\left(h^{A-} * h^{B}\right)\right\rangle(t, \boldsymbol{x}) \mathrm{dS} .
\end{aligned}
$$

Here, $f * g$ is the convolution of $f$ and $g$ with respect to time (thus $f * g=g * f$ ):

$$
f * g(t, \cdot) \equiv \int_{0}^{t} f(r, \cdot) g(t-r, \cdot) \mathrm{d} r
$$

This is an extension of Proposition 1 to unsteady problems for fixed bounded domains. The symmetry of the roles of problems A and B is retained thanks to the symmetric property of convolution. From the relation (14), one can derive a natural extension of the flux representations to unsteady problems. The reader is referred to Sec. 5 of Ref. [12] for the missing details. Here, we shall present general representation of mass, momentum, and energy in the domain by considering the response of the system to the initial perturbation, which is peculiar to unsteady problems, and to "external force." Then, the Poiseuille flow and thermal transpiration are discussed in view of the obtained representations.

\footnotetext{
${ }^{6}$ The appropriateness of the kinetic boundary conditions in view of the thermodynamics was studied by L. Waldmann, I. Kuščer, etc. See, for example, Refs. $[1,5,8]$ and references therein.
} 
Let us denote by $G^{(\alpha)}$ the response of the system to spatially uniform initial data $\phi_{\text {initial }}=\alpha(\zeta)$, where $\alpha$ is an arbitrary given function. Thus, $G^{(\alpha)}$ is a solution of initial- and boundary-value problem (12), (13), (2)-(3b) with $g_{\mathrm{w}}=h=I=0$ and $\phi_{\text {initial }}=\alpha$ (i.e., $G_{\text {initial }}^{(\alpha)}=\alpha$ ). Then, from (14), we obtain the following:

Proposition 3 Consider the initial-and boundary-value problem (12), (13), (2)-(3b) in the domain D. Then the total $\alpha$-moment in the domain at any time are expressed in terms of the Green function for the initial data $\alpha$ :

$$
\begin{aligned}
\operatorname{Sh} \int_{D}\left\langle\alpha^{-} \phi\right\rangle(t, \boldsymbol{x}) \mathrm{d} \boldsymbol{x}=\operatorname{Sh} \int_{D}\left\langle\phi_{\text {initial }}^{-} G^{(\alpha)}\right\rangle(t, \boldsymbol{x}) \mathrm{d} \boldsymbol{x}+\int_{D}\left\langle I^{-} * G^{(\alpha)}\right\rangle(t, \boldsymbol{x}) \mathrm{d} \boldsymbol{x} & \\
& -\int_{\partial D_{\mathrm{w}}}\left\langle\zeta_{i} n_{i}\left(g_{\mathrm{w}}^{-} * G^{(\alpha)}\right)\right\rangle(t, \boldsymbol{x}) \mathrm{dS}-\int_{\partial D_{\mathrm{g}}}\left\langle\zeta_{i} n_{i}\left(h^{-} * G^{(\alpha)}\right)\right\rangle(t, \boldsymbol{x}) \mathrm{dS} .
\end{aligned}
$$

When $\alpha=1, \zeta_{i}$, and $\frac{2}{3}|\zeta|^{2}$, (15) gives the expression of the total mass, momentum, and energy in the domain.

Poiseuille flow and thermal transpiration. Consider the time-dependent Poiseuille flow and thermal transpiration (Fig. 1). The former can be formulated as the flow of a gas, which is initially in the thermal equilibrium at rest with the pipe wall of uniform temperature, caused by a uniform weak external force in the (negative) $x_{1}$-direction. Thus, $\phi^{\mathrm{P}}$ is a solution of (12), (13), and (2) with $D=\mathrm{S}, \partial D=\partial D_{\mathrm{w}}=\partial \mathrm{S}, I=-\zeta_{1}, g_{\mathrm{w}}=0$, and $\phi_{\text {initial }}^{\mathrm{P}}=0$. On the other hand, the solution $\phi^{\mathrm{T}}$ of the thermal transpiration problem can be expressed as $\phi^{\mathrm{T}}=x_{1}\left(|\boldsymbol{\zeta}|^{2}-\frac{5}{2}\right)+\Phi^{\mathrm{T}}\left(t, \boldsymbol{x}_{\perp}, \boldsymbol{\zeta}\right)$, where $\Phi^{\mathrm{T}}$ is a solution of (12), (13), and (2) with $D=\mathrm{S}, \partial D_{\mathrm{w}}=\partial \mathrm{S}, \partial D_{\mathrm{g}}=0, I=-\zeta_{1}\left(|\boldsymbol{\zeta}|^{2}-\frac{5}{2}\right), g_{\mathrm{w}}=0$, and a certain initial data $\Phi_{\text {initial }}^{\mathrm{T}}\left(\boldsymbol{x}_{\perp}, \boldsymbol{\zeta}\right)$. Then, from (15) with $\alpha=\zeta_{1}$, the following expressions are obtained:

$$
\begin{aligned}
& \operatorname{Sh} \int_{\mathrm{S}}\left\langle\zeta_{1} \phi^{\mathrm{P}}\right\rangle\left(t, \boldsymbol{x}_{\perp}\right) \mathrm{d} \boldsymbol{x}_{\perp}=-\int_{0}^{t} \int_{\mathrm{S}}\left\langle\zeta_{1} G^{\left(\zeta_{1}\right)}\right\rangle\left(s, \boldsymbol{x}_{\perp}\right) \mathrm{d} \boldsymbol{x}_{\perp} \mathrm{d} s, \\
& \operatorname{Sh} \int_{\mathrm{S}}\left\langle\zeta_{1} \phi^{\mathrm{T}}\right\rangle\left(t, \boldsymbol{x}_{\perp}\right) \mathrm{d} \boldsymbol{x}_{\perp}=-\operatorname{Sh} \int_{\mathrm{S}}\left\langle\Phi_{\text {initial }}^{\mathrm{T}-} G^{\left(\zeta_{1}\right)}\right\rangle\left(t, \boldsymbol{x}_{\perp}\right) \mathrm{d} \boldsymbol{x}_{\perp}-\int_{0}^{t} \int_{\mathrm{S}}\left\langle\zeta_{1}\left(|\boldsymbol{\zeta}|^{2}-\frac{5}{2}\right) G^{\left(\zeta_{1}\right)}\right\rangle\left(s, \boldsymbol{x}_{\perp}\right) \mathrm{d} \boldsymbol{x}_{\perp} \mathrm{d} s,
\end{aligned}
$$

where the relation $u_{1}\left[\phi^{\mathrm{T}}\right]=u_{1}\left[\Phi^{\mathrm{T}}\right]$ has been taken into account. Thus, the mass flow (flux) through the pipe can be expressed for any time in terms of the Green function for the initial data $\zeta_{1}$. Further, since $G^{\left(\zeta_{1}\right)} \rightarrow 0$ as $t \rightarrow \infty$, (16) is reduced in the same limit to

$$
\begin{aligned}
& \text { Sh } \int_{\mathrm{S}}\left\langle\zeta_{1} \phi^{\mathrm{P}}\right\rangle\left(\boldsymbol{x}_{\perp}\right) \mathrm{d} \boldsymbol{x}_{\perp}=-\int_{0}^{\infty} \int_{\mathrm{S}}\left\langle\zeta_{1} G^{\left(\zeta_{1}\right)}\right\rangle\left(s, \boldsymbol{x}_{\perp}\right) \mathrm{d} \boldsymbol{x}_{\perp} \mathrm{d} s, \\
& \operatorname{Sh} \int_{\mathrm{S}}\left\langle\zeta_{1} \phi^{\mathrm{T}}\right\rangle\left(\boldsymbol{x}_{\perp}\right) \mathrm{d} \boldsymbol{x}_{\perp}=-\int_{0}^{\infty} \int_{\mathrm{S}}\left\langle\zeta_{1}\left(|\boldsymbol{\zeta}|^{2}-\frac{5}{2}\right) G^{\left(\zeta_{1}\right)}\right\rangle\left(s, \boldsymbol{x}_{\perp}\right) \mathrm{d} \boldsymbol{x}_{\perp} \mathrm{d} s .
\end{aligned}
$$

The left-hand side is the mass flow of the steady Poiseuille flow and thermal transpiration. These expressions will be interpreted as an extension of the fluctuation-dissipation theorem to the case of arbitrary $\mathrm{Kn}$ in the next section.

Next we consider the response of the system to an "external force" $I=\alpha(\boldsymbol{\zeta})$, which is uniform both in time and space, and denote it by $G^{(\alpha ; I)}$, where $\alpha$ is an arbitrary function. Thus, $G^{(\alpha ; I)}$ is a solution of initial- and boundary-value problem (12), (13), (2)-(3b) with $g_{\mathrm{w}}=h=\phi_{\text {initial }}=0$ and $I=\alpha$. Then, from (14), we obtain the following:

Proposition 4 Consider the initial-and boundary-value problem (12), (13), (2)-(3b) in the domain D. Then, the total $\alpha$-moment in the domain from the initial time can be expressed in terms of the Green function for "external force" $\alpha$ :

$$
\begin{aligned}
\int_{0}^{t} \int_{D}\left\langle\alpha^{-} \phi\right\rangle(s, \boldsymbol{x}) \mathrm{d} \boldsymbol{x} \mathrm{d} s=\operatorname{Sh} \int_{D}\left\langle\phi_{\text {initial }}^{-} G^{(\alpha ; I)}\right\rangle(t, \boldsymbol{x}) \mathrm{d} \boldsymbol{x}+\int_{D}\left\langle I^{-} * G^{(\alpha ; I)}\right\rangle(t, \boldsymbol{x}) \mathrm{d} \boldsymbol{x} \\
\quad-\int_{\partial D_{\mathrm{w}}}\left\langle\zeta_{i} n_{i}\left(g_{\mathrm{w}}^{-} * G^{(\alpha ; I)}\right)\right\rangle(t, \boldsymbol{x}) \mathrm{dS}-\int_{\partial D_{\mathrm{g}}}\left\langle\zeta_{i} n_{i}\left(h^{-} * G^{(\alpha ; I)}\right)\right\rangle(t, \boldsymbol{x}) \mathrm{dS} .
\end{aligned}
$$

When $\alpha=1, \zeta_{i}$, and $\frac{2}{3}|\zeta|^{2}$, (17) gives the expression of total mass, momentum, and energy in the domain from the initial time. 
Poiseuille flow and thermal transpiration. Consider again the time-dependent Poiseuille flow and thermal transpiration problems. As is seen from its definition, $\phi^{\mathrm{P}}$ is no other than $G^{\left(-\zeta_{1} ; I\right)}\left(=-G^{\left(\zeta_{1} ; I\right)}\right)$. Thus, (17) with $\alpha=-\zeta_{1}$ and $\phi=\Phi^{\mathrm{T}}$ eventually leads to

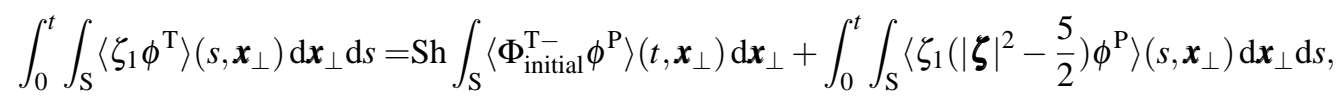

and thus

$$
\int_{\mathrm{S}}\left\langle\zeta_{1} \phi^{\mathrm{T}}\right\rangle\left(t, x_{\perp}\right) \mathrm{d} x_{\perp}=\operatorname{Sh} \partial_{t} \int_{\mathrm{S}}\left\langle\Phi_{\text {initial }}^{\mathrm{T}-} \phi^{\mathrm{P}}\right\rangle\left(t, x_{\perp}\right) \mathrm{d} x_{\perp}+\int_{\mathrm{S}}\left\langle\zeta_{1}\left(|\zeta|^{2}-\frac{5}{2}\right) \phi^{\mathrm{P}}\right\rangle\left(t, x_{\perp}\right) \mathrm{d} x_{\perp} .
$$

Equation (18b) is an extension of the reciprocal relation between the steady Poiseuille flow and thermal transpiration. In the limit $t \rightarrow \infty$, the first term on the right-hand side vanishes, because $\phi^{\mathrm{P}}$ tends to a steady solution. Thus, in the same limit, (18b) is reduced to the known reciprocity relation (11). [The present $\phi^{\mathrm{P}}$ with $t \rightarrow \infty$ is different from $\phi^{\mathrm{P}}$ in (11) by $x_{1}$. However, this difference does not change the form of the relation (11).] It should be noted that the same reciprocity as the steady case remains valid for any time, if $\Phi_{\text {initial }}^{\mathrm{T}}=0$.

\section{SIMILARITY TO FLUCTUATION-DISSIPATION THEOREM}

The Green functions $G^{(\alpha)}$ and $G^{(\alpha ; I)}$ in the previous section are closely related to each other. In fact, it is confirmed by substitution that $\mathrm{Sh}^{-1} \int_{0}^{t} G^{(\alpha)}(s, \boldsymbol{x}, \boldsymbol{\zeta}) \mathrm{d} s$ solves the initial- and boundary-value problem for $G^{(\alpha ; I)}$ :

$$
G^{(\alpha ; I)}(t, \boldsymbol{x}, \boldsymbol{\zeta})=\mathrm{Sh}^{-1} \int_{0}^{t} G^{(\alpha)}(s, \boldsymbol{x}, \boldsymbol{\zeta}) \mathrm{d} s .
$$

Thus, for two arbitrary functions $\alpha(\boldsymbol{\zeta})$ and $\beta(\boldsymbol{\zeta})$, the following identity holds:

$$
\int_{D}\left\langle\beta G^{(\alpha ; I)}\right\rangle(t, x) \mathrm{d} \boldsymbol{x}=\mathrm{Sh}^{-1} \int_{0}^{t} \int_{D}\left\langle G_{\text {initial }}^{(\beta)} G^{(\alpha)}\right\rangle(s, \boldsymbol{x}) \mathrm{d} \boldsymbol{x} \mathrm{d} s
$$

Equation (19) may be regarded as the counterpart of the fluctuation-dissipation theorem for bulk systems or as its extension to the system of arbitrary Knudsen number (non-bulk system), which is understood as follows:

(i) Put $\alpha=\beta$ and take the limit $t \rightarrow \infty$. Then, the left-hand side of (19) represents the system response to "external force" $\alpha$, while the right-hand side represents the self-correlation of the relaxation process from initial data $\alpha$.

(ii) Applying (14) to the pair of $G^{(\alpha)}$ and $G^{(\beta)}$ gives

$$
\int_{D}\left\langle G_{\text {initial }}^{(\beta)-} G^{(\alpha)}\right\rangle(t, x) \mathrm{d} \boldsymbol{x}=\int_{D}\left\langle G_{\text {initial }}^{(\alpha)-} G^{(\beta)}\right\rangle(t, x) \mathrm{d} \boldsymbol{x} .
$$

Thus, if $\alpha$ and $\beta$ are even or odd with respect to $\zeta$, we obtain from (19) and (20)

$$
\int_{D}\left\langle\beta G^{(\alpha ; I)}\right\rangle(t, \boldsymbol{x}) \mathrm{d} \boldsymbol{x}=\varepsilon_{\alpha} \varepsilon_{\beta} \int_{D}\left\langle\alpha G^{(\beta ; I)}\right\rangle(t, \boldsymbol{x}) \mathrm{d} \boldsymbol{x},
$$

where $\varepsilon_{\alpha}\left(\right.$ or $\left.\varepsilon_{\beta}\right)=1$ if $\alpha$ (or $\beta$ ) is even and -1 if $\alpha$ (or $\beta$ ) is odd. In the limit $t \rightarrow \infty$, the integrand of both sides of (21) represents the static admittance at $\boldsymbol{x}$. In this sense, (21) represents an extension of the symmetry of static admittance $[17,18]$ to the system of arbitrary Kn.

It should be noted that the above approach is more direct and contains a little stronger consequences than that of Sec. 6 in Ref. [12]. We shall show below two examples that supplement the above observations.

Response of the gas in a periodic box. Let $D$ be a periodic box and consider the system response $G^{\left(2 \zeta_{1} \zeta_{2} ; I\right)}$ against the "external force" $\alpha=2 \zeta_{1} \zeta_{2}$. Obviously $G^{\left(2 \zeta_{1} \zeta_{2} ; I\right)}$ is spatially uniform and $G^{\left(2 \zeta_{1} \zeta_{2} ; I\right)} \rightarrow(\sqrt{\pi} / 2) \zeta_{1} \zeta_{2} B(|\zeta|)$ as $t \rightarrow \infty$. Here, $B$ is the solution of $\mathscr{L}\left(\zeta_{1} \zeta_{2} B\right)=-2 \zeta_{1} \zeta_{2}$. It is well-known that $B$ gives the viscosity $\mu$ [16]: $\mu=\sqrt{\pi} p_{0}\left(2 k T_{0} / m\right)^{-1 / 2} \ell_{0}\left\langle\zeta_{1}^{2} \zeta_{2}^{2} B\right\rangle$. Now, as in (i), we take the limit $t \rightarrow \infty$ in (19) with $\alpha=\beta=2 \zeta_{1} \zeta_{2}$ to have

$$
\mu=p_{0} t_{0} \int_{0}^{\infty}\left\langle G_{\text {initial }}^{\left(2 \zeta_{1} \zeta_{2}\right)} G^{\left(2 \zeta_{1} \zeta_{2}\right)}(t, \zeta)\right\rangle \mathrm{d} t
$$


In the same way, by considering the system response $G^{\left(2 \zeta_{1} \zeta_{2} ; I\right)}$ against the "external force" $\alpha=\zeta_{1}\left(|\zeta|^{2}-\frac{5}{2}\right)$, we eventually obtain the expression of thermal conductivity $\lambda$ from (19):

$$
\lambda=2(k / m) p_{0} t_{0} \int_{0}^{\infty}\left\langle G_{\text {initial }}^{\left(\zeta_{1}\left(|\zeta|^{2}-\frac{5}{2}\right)\right)} G^{\left(\zeta_{1}\left(|\zeta|^{2}-\frac{5}{2}\right)\right)}(t, \zeta)\right\rangle \mathrm{d} t
$$

These correspond to the so-called Kubo formula [17] in the linear response theory.

Poiseuille flow and thermal transpiration. We discuss again the time-dependent Poiseuille flow and thermal transpiration and give an alternative interpretation to the formulas in the previous sections. As already mentioned, $\phi^{\mathrm{P}}=G^{\left(-\zeta_{1} ; I\right)}\left(=-G^{\left(\zeta_{1} ; I\right)}\right)$. Thus the expression (16a) is no other than (19) with $\alpha=\beta=\zeta_{1}$. Further, because $\Phi^{\mathrm{T}}$ is written as $\Phi^{\mathrm{T}}=G^{\left(\Phi_{\text {initial }}^{\mathrm{T}}\right)}+G^{\left(-\zeta_{1}\left(|\zeta|-\frac{5}{2}\right) ; I\right)}\left(=G^{\left(\Phi_{\text {initial }}^{\mathrm{T}}\right)}-G^{\left(\zeta_{1}\left(|\zeta|-\frac{5}{2}\right) ; I\right)}\right)$, the expression (18a) with $\Phi_{\text {initial }}^{\mathrm{T}}=0$ is no other than (21) with $\alpha=\zeta_{1}$ and $\beta=\zeta_{1}\left(|\zeta|^{2}-\frac{5}{2}\right.$ ). Thus, the expressions (16a) and (18a) may be regarded as the extensions of the fluctuation-dissipation theorem and of the symmetry of static admittance respectively. In particular, if we take the limit $t \rightarrow \infty$ for the latter, the reciprocity between the steady Poiseuille flow and thermal transpiration is recovered. This is an alternative explanation of the known reciprocity relation by the present approach.

\section{CONCLUDING REMARKS}

In this paper, we discussed the relations between two independent problems from the viewpoint of Green functions. The present approach based on this viewpoint enabled us to establish the reciprocity relation in a pointwise way (Green reciprocity), by which a general representation of fluxes is deduced. It further enabled us to develop the theory for steady problems to unsteady problems. As a result, we obtained a natural extension (or counterpart) of the consequences of the linear response theory for bulk system to the system of arbitrary $\mathrm{Kn}$.

It is straightforward to develop the present theory $[11,14,12]$ to gas mixtures. However, it should be noted that, in order to obtain the result for unbounded domain corresponding to Refs. [11,14], it is necessary to prove a lemma corresponding to lemma 2 of Ref. [11]. This can be done by using that in a far field the concentration of component gas obeys the Laplace equation, in addition to that the flow velocity and temperature behave in the same way as in the case of single-component gas.

\section{ACKNOWLEDGMENTS}

The present work is supported in part by KAKENHI (No. 19560066) from JSPS.

\section{REFERENCES}

1. L. Waldmann, Z. Naturforsch 22a, 1269-1280 (1967).

2. H. Lang, Phys. Fluids 19, 366-371 (1976).

3. S. K. Loyalka, I. J. Chem. Phys. 55, 4497-4503 (1971).

4. B. I. M. ten Bosch, J. J. M. Beenakker, and I. Kuščer, Physica 123A, 443-462 (1984).

5. F. R. W. McCourt, J. J. M. Beenakker, W. E. Köhler, and I. Kuščer, Nonequilibrium Phenomena in Polyatomic Gases, Vol. 2 , Clarendon, Oxford, 1991.

6. V. I. Roldughin, J. Non-Equilib. Thermodyn. 19, 349-367 (1994).

7. F. Sharipov, Physica A 203, 437-456 (1994).

8. V. M. Zhdanov, V. I. Roldughin, Physics Uspekhi 41, 349-378 (1998).

9. V. I. Roldughin, V. M. Zhdanov, Advances in Colloid and Interface Science 98, 121-215 (2002).

10. F. Sharipov, Phys. Rev. E 73, 026110 (2006).

11. S. Takata, J. Stat. Phys. 136, 751-784 (2009). ( $\frac{5}{2} \gamma_{2}$ on line 18, p. 762 and line 24, p. 763 in this reference is a misprint of $\left.\frac{5}{4} \gamma_{2}.\right)$

12. S. Takata, J. Stat. Phys. 140, 985-1005 (2010).

13. S. Takata, Phys. Fluids 21, 112001 (2009).

14. S. Takata, J. Stat. Phys. 136, 945-983 (2009).

15. C. Cercignani, The Boltzmann Equation and its Applications, Springer, New York, 1988.

16. Y. Sone, Molecular Gas Dynamics, Birkhäuser, Boston, 2007 (Suppl. Notes \& Errata: http://hdl.handle.net/2433/66098).

17. R. Kubo, M. Toda, and N. Hashitsume, Statistical Physics II, Nonequilibrium Statistical Mechanics, 2nd ed., Springer, Berlin, 1991, Chap. 4.

18. N. Pottier, Nonequilibrium Statistical Physics, Oxford University Press, New York, 2010. 
Copyright of AIP Conference Proceedings is the property of American Institute of Physics and its content may not be copied or emailed to multiple sites or posted to a listserv without the copyright holder's express written permission. However, users may print, download, or email articles for individual use. 